\title{
Multi-Mode Antenna Feed for Ultra Wideband Technology
}

\author{
Grzegorz Adamiuk, Werner Wiesbeck, Thomas Zwick \\ Institut für Höchstfrequenztechnik und Elektronik, University of Karlsruhe, Kaiserstr. 12, 76128 \\ Karlsruhe, Germany, mail: grzegorz.adamiuk@ihe.uka.de
}

\begin{abstract}
This paper describes the principle and design of a broadband feed for multi mode antennas designated for Ultra Wideband (UWB) technique. The feed consists of slotlines, which are excited in two different modes: coplanar waveguide (CPW) and coupled slot-lines (CSL) mode. Each of the modes is excited by a separate connector. Therefore, different radiation patterns for each mode result. In order to show the feasibility of such an approach the feed is integrated into tapered slot antennas. Subsequently measured radiation patterns and time domain properties of such an antenna are given.
\end{abstract}

Index Terms - Antennas, Antenna Feeds, Antenna Radiation Patterns, Ultra Wideband.

\section{INTRODUCTION}

Ultra Wideband is one of the emerging technologies that has gained the attention of scientists and industry worldwide. Due to its enormous bandwidth UWB has a big potential in almost all microwave systems from telecommunication to radar applications. The advantages occur, dependent on application, in the high data rates, very high resolution or very fine localization possibilities. A very challenging topic is the design of antennas for UWB covering the desired bandwidth, which extends in many applications from $3.1 \mathrm{GHz}$ to $10.6 \mathrm{GHz}$. A very good candidate for such an antenna is a tapered slot antenna, already introduced in numerous publications [1], [2].

For certain applications such as radar, localization, MIMO the antennas with a reconfigurable radiation pattern are desired [3]. Also a direction finding with one single multimode antenna is possible [4]. Such antennas can be realized by use of a multimode feed. Different guided modes within a feed cause different electric field distributions in the antenna and thus change its radiation pattern [5]. A challenging task is therefore how to realize an antenna feed, which is able to guide different modes in the same structure and easy to integrate in a compact, planar antenna structure. However, an equally important task is the design of an antenna itself. Such an antenna has to be able to take an advantage of a multimode feed, the input impedance must be able to be matched to the impedances of two different modes, and has to radiate over a very wide bandwidth with acceptable time domain behavior [2].

\section{ANTENNA FEED}

As base structure for guiding two different modes two slot-lines placed near to each other were chosen. They can be driven generally in two modes: coplanar waveguide (CPW) and coupled slot-line (CSL) mode. The electric field distribution in a cross section of the feed driven in these two different modes is introduced in Fig. 1. It can be observed that the electric field vectors in different modes are oriented differently to each other. These two modes fed to the antenna cause different electric field distributions in the antenna and therefore radiation in different directions is possible.
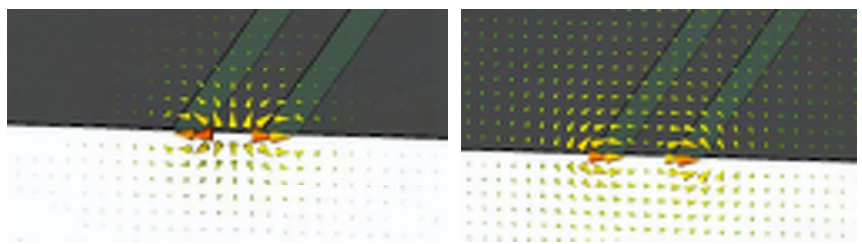

Fig. 1 CPW (left) and CSL (right) mode in two slot-lines placed near to each other.

The task to solve is the proper excitation of these two modes. In our approach two different connectors for each mode are used. A requirement is however to excite each mode independently and with small coupling between the two connectors. Such a solution was presented in [6]. However the proposed structure was hard to optimize for the whole desired bandwidth and was sensible to the mechanical tolerances. Therefore, in this paper, a new antenna feed overcoming these drawbacks is presented. A schematic of the structure is shown in Fig. 2. Port 3 is the part of the feed that is connected directly to the antenna.

A CPW mode is created by directly soldering the connector to the waveguide. The inner conductor, on the other hand, is connected to the middle line, whereas the side metallization is connected to the outer conductor of the connector. In Fig. 2 the CPW mode is excited at port 1.

A CSL mode is excited by an aperture coupling of the wave guided in a micro-strip line. The signal is coupled to the slots simultaneously, which results in both slots guiding the same signal and the electric field vectors having the same orientation. A shortcut near the place of 

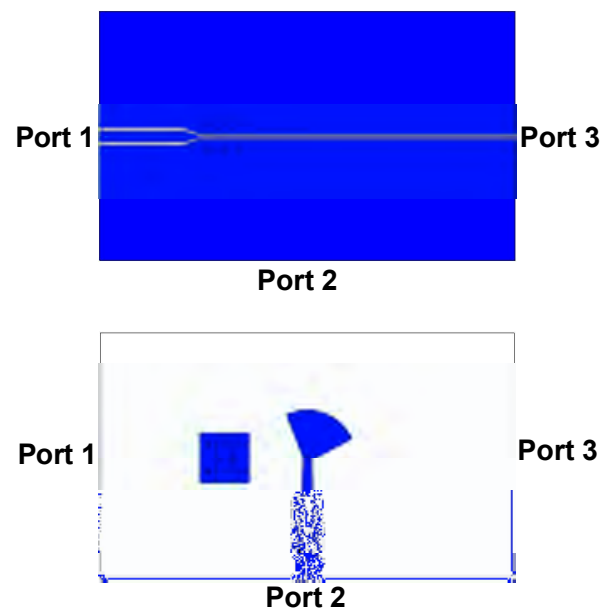

Fig. 2 Top and bottom view of a feed for a broadband multi mode antenna (Port 1 - CPW mode, Port 2 - CSL mode, Port 3 - antenna connection).

the coupling prevents a coupling of the signal to the other connector. The shortcut is realized as a set of vias that are connected by a metallization on the back side of the feed. The port for the excitation of the CSL mode is marked in Fig. 2 as port 2. This principle is similar to the one for typical aperture coupling from micro-strip line to slotline [2].

The structure was simulated in the commercial simulation tool CST Microwave Studio [7]. The relevant simulated S-Parameters are shown in Fig. 3. It can be noticed that in the whole desired bandwidth the matching at the ports 1 and 2 is better than $-10 \mathrm{~dB}$. The coupling factors $S_{31}$ and $S_{32}$ show relative high values, which stand for high transmission of the energy from the feeding ports to the antenna. The decoupling $\mathrm{S}_{21}$ between the feeding ports has a value significantly larger than $20 \mathrm{~dB}$ and therefore is not shown in the diagram.

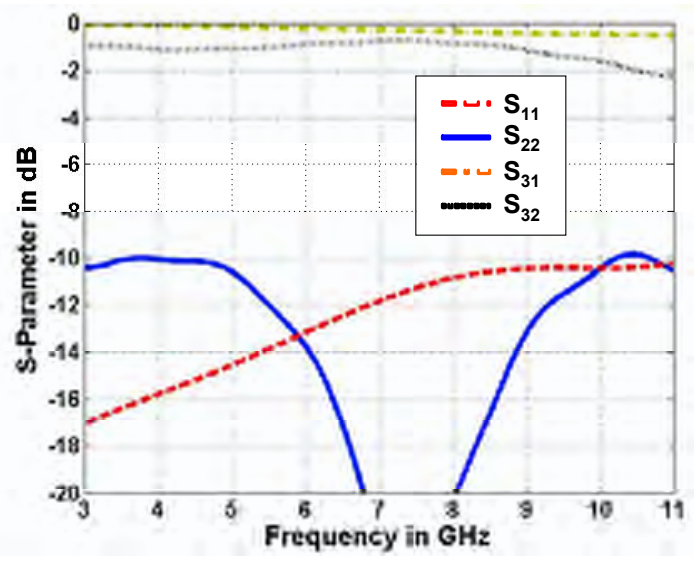

Fig. 3 Simulated S-Parameters of the broadband feed for multi mode antennas (notation as in Fig. 2).

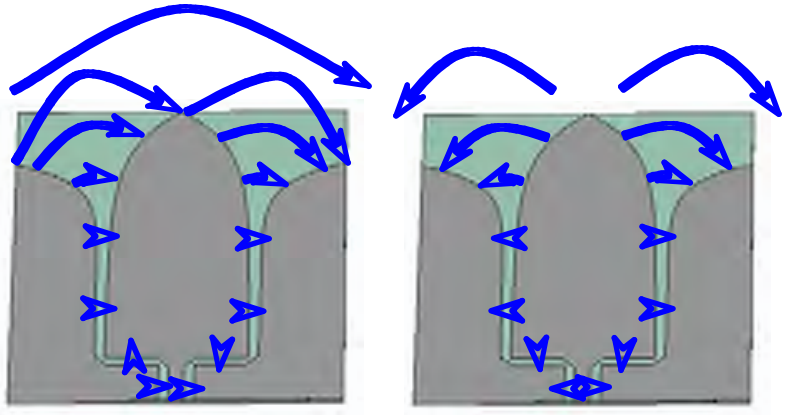

Fig. 4 Creation of a radiation pattern of the antenna fed by two slot-lines driven in CSL (left) and CPW (right) mode.

\section{PRACTICAL VERIFICATION}

In the literature [6] a spiral antenna has been used as a broadband radiating element able to profit from multi mode feed. However a spiral antenna has a too long impulse response and is thus not a perfect candidate for the ultra wideband technique [1]. Therefore two tapered slot antennas were used instead. Each antenna was connected to the single slot at the output of the feed. With this configuration the feeding with the CPW and CSL mode creates two different radiation patterns. A principle of the radiation is shown in Fig. 4. In CSL mode the one single beam is created, which results from superposition of the fields radiated from the single slots.
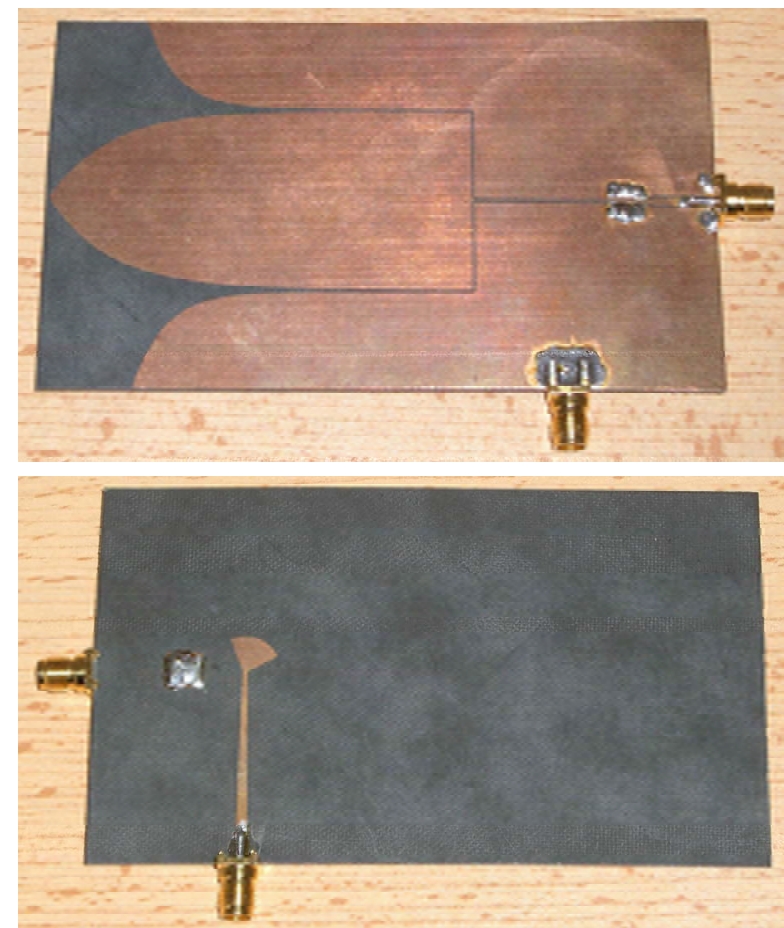

Fig. 5 Realization of the UWB antenna with multi mode feed (top and bottom view). 
In CPW mode, on the other hand, two beams are created, which are oriented to the sides. The minimum of the radiation is oriented in the direction of the main beam in the CSL mode.

Such an antenna was realized on the substrate Duroid 5880 with the relative permittivity of 2.2 and thickness $0.79 \mathrm{~mm}$. The photo of the whole structure can be seen in Fig. 5. On the right side the port for the excitation of the CPW mode can be seen. On the bottom of the antenna a port for the CSL mode can be seen. Two tapered slots are placed on the left side. With the shape of the slots and the distance to each other the radiation pattern in both modes can be influenced. In the following section the measurement results of the presented device are given.

\section{Results}

The measurement of the input impedance matching showed a very broadband behavior between $3.1 \mathrm{GHz}$ and 10.6 GHz. The S-Parameters posses several minima and maxima due to resonances, which resulted in the worse matching factor at particular frequencies. It is due to the fact that the multi-mode waveguides possess different input impedances for different modes, the optimization becomes difficult. However $S_{11}$ and $S_{22}$ were lower than $-6 \mathrm{~dB}$ for almost the whole desired bandwidth for both modes. Since the power in UWB is very low, the mismatching at the antenna input will not affect the frontend electronics.

The measurements of the coupling factors $S_{21}$ between both ports of the antenna showed high decoupling. The value was better than $20 \mathrm{~dB}$ in the whole desired frequency range.

The radiation characteristics of the antenna were measured in an anechoic chamber. For the measurements a vector network analyzer was used. Complex transfer characteristics allowed calculation of the amplitude and phase behavior of the antenna, which is needed for the antenna characterization in the time domain. Characterization by the impulse response is very helpful in the description of the behavior of the device in pulsemode operation.

During the measurements a $50 \mathrm{Ohm}$ terminator was connected to the port of the antenna, which was not used.

Since the multi-mode characteristics of the presented antenna can be observed clearly only in the E-Plane, in the following the measurement and simulation results of the E-Plane of the antenna are presented.

In Fig. 6 (left) and Fig. 7 (left) the measured gain over frequency and angle of the antenna fed at port 3 (CPW mode) is presented. As expected the antenna radiates in

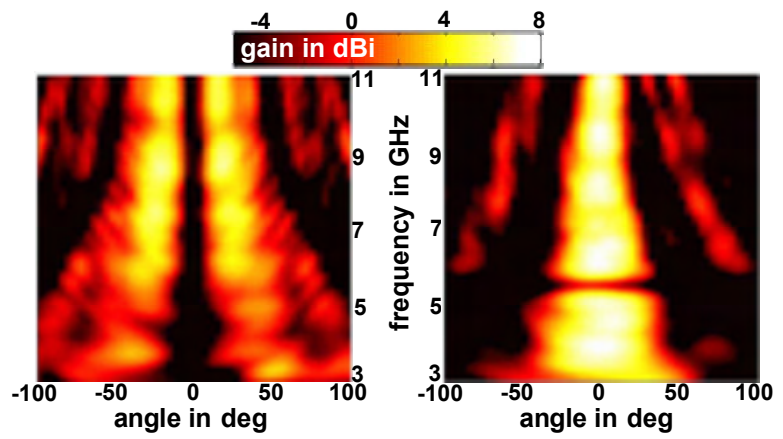

Fig. 6 Measured gain (E-Plane) over frequency and angle of the antenna in Fig. 5 excited by the CPW (left) and CSL (right) mode.

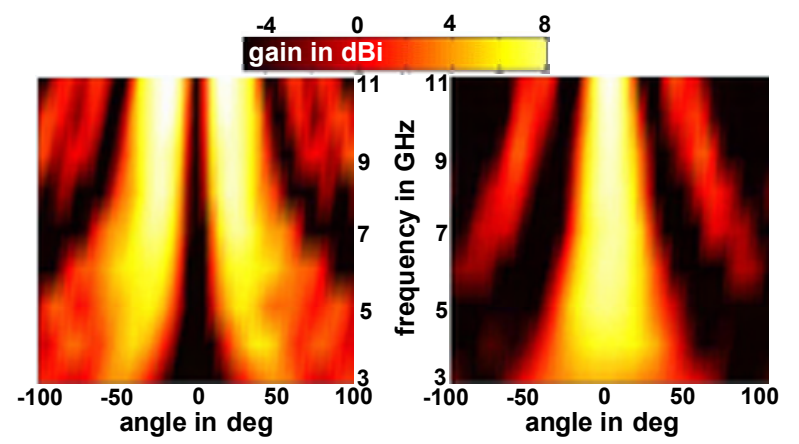

Fig. 7 Simulated gain (E-Plane) over frequency and angle of the antenna in Fig. 5 excited by the CPW (left) and CSL (right) mode.

CPW mode with two beams with minimum radiation at 0 degrees. A maximal gain of approx. $4 \mathrm{dBi}$ was measured. The maxima of both beams point the direction of approx. +/-45 degrees.

In Fig. 6 (right) and Fig. 7 (right) the measured and simulated gain of the antenna fed at port 2 (CSL mode) is presented, respectively. In contrast to the gain measurements in the CPW mode, maximal gain is at 0 degrees and is relatively symmetric. A slight squint of the beam with the frequency occurs due to uneven excitation of both feeding slots. Since the squint is very weak, it does not have a big influence on the antenna performance.

The beam is relatively concentrated. The maximal achieved gain was over $7 \mathrm{dBi}$. The values of the gain vary slightly over frequency, which is due to mismatching of the antenna at particular frequencies. However, this fact does not have a strong influence on the overall performance, since the gain is still relatively high. Merely the mismatching at approx. $5.5 \mathrm{GHz}$ can be clearly seen in the gain measurements. But for the pulsemode operation it is of small interest, since the impulse response of the antenna does not exhibit negative properties. In the following the time domain properties of the antenna are presented. 
From an UWB antenna a short impulse response is expected. The shorter the impulse response the less dispersed the radiated pulse is. Furthermore the amplitude of the impulse response gives the energy concentration in a specified direction and can be related to the gain in the frequency domain. The other parameter characterizing an antenna in the time domain is the ringing time of the impulse response. It describes the oscillations of the radiated pulse after the main peak and is expected to be as short as possible.

In Fig. 8 the impulse response of the antenna fed at the port 3 (CPW mode) is presented. It can be noticed that the impulse response is very concentrated in time. The amplitude distribution is analog to the gain measurements. The minimum is located at 0 degrees and is symmetric. The ringing of the impulse response in CPW mode is very weak.

In Fig. 9 the impulse response of the antenna fed in CSL mode is depicted. It is similar to the single, aperture coupled Vivaldi antenna. The maximum of the amplitude occurs at 0 degrees. The length of the impulse response is slightly longer than the one presented in Fig. 8. This is due to the aperture coupling, which differentiates the signal and makes the impulse response longer. The ringing in the presented mode is also stronger. However the time domain behavior of the antenna fed by the CSL mode is still very advantageous and can be successfully applied for the pulse-mode operation.

\section{CONCLUSIONS}

In this paper a multi-mode antenna feed with an aperture coupling for the ultra wideband technology was presented. The device exhibits very good matching and decoupling in the frequency range between $3.1 \mathrm{GHz}$ and 10.6 GHz. In order to show the feasibility of the feed, a prototype of multi mode antenna for UWB technique was built. The measured values complied well with simulation data. For each of the modes typical radiation characteristics were observed: a single beam for CSL mode and two, split beams for the CPW mode. The time domain properties have confirmed a good applicability of the antenna in pulse based ultra wideband systems.

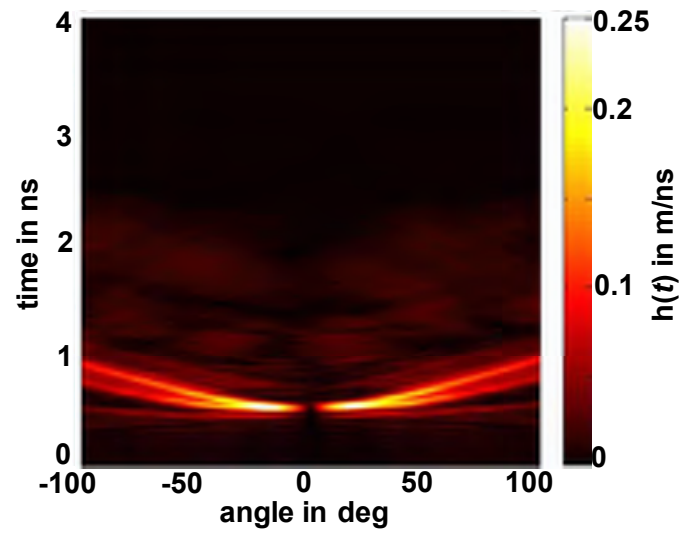

Fig. 8 Measured impulse response $\mathrm{h}(t)$ (E-Plane) over time and angle of the antenna in Fig. 5 excited by an odd mode.

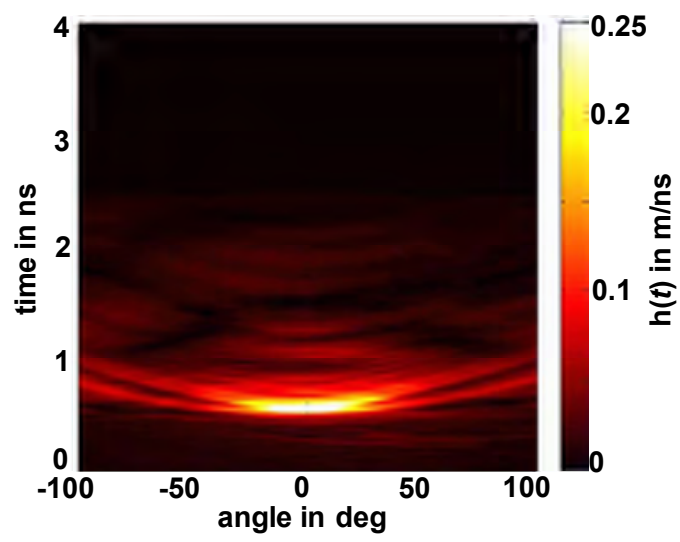

Fig. 9 Measured impulse response $\mathrm{h}(t)$ (E-Plane) over time and angle of the antenna in Fig. 5 excited by an even mode.

\section{REFERENCES}

[1] W. Wiesbeck, G. Adamiuk, „Antennas for UWB-Systems“, 2nd International ITG Conference on Antennas, 2007. INICA '07, pp. 67-71, 28-30 March 2007

[2] W. Sörgel, W. Wiesbeck, "Influence of the Antennas on the Ultra Wideband Transmission", EURASIP Journal on Applied Signal Processing, special issue UWB - State of the Art, pp. 296305, March 2005

[3] Svantesson, T, "Correlation and channel capacity of MIMO systems employing multimode antennas", IEEE Transactions on Vehicular Technology, vol. 51, Issue 6, pp:1304 - 1312, Nov. 2002

[4] Svantesson, T., "Multimode based direction finding", Signals, Systems and Computers, 2000. Conference Record of the ThirtyFourth Asilomar Conference on, vol. 1, pp:595-599, 29 Oct.-1 Nov. 2000

[5] Stuart, Howard R.; Best, Steven R. "A small wideband multimode antenna" Antennas and Propagation Society International Symposium, 2008. AP-S 2008. IEEE, pp:1-4, 5-11 July 2008

[6] E. Gschwendtner, W. Wiesbeck, „Ultra-broadband car antennas for communications and navigation applications", IEEE Transactions on Antennas and Propagation, Volume 51, Issue 8, pp. 2020-2027, Aug. 2003

[7] CST Microwave Studio ${ }^{\circledR}$, www.cst.com 